\title{
An Enhanced Secondary Control Approach for Voltage Restoration in the DC Distribution System
}

\author{
Author, co-author
}

Affiliation

\begin{abstract}
The paper will deal with the problem of establishing a desirable power sharing in multi-feed electric power system for future moreelectric aircraft (MEA) platforms. The MEA is one of the major trends in modern aerospace engineering aiming for reduction of the overall aircraft weight, operation cost and environmental impact. Electrical systems are employed to replace existing hydraulic, pneumatic and mechanical loads. Hence the onboard installed electrical power increases significantly and this results in challenges in the design of electrical power systems (EPS). One of the key paradigms for future MEA EPS architectures assumes high-voltage dc distribution with multiple sources, possibly of different physical nature, feeding the same bus(es). In our study we investigate control approaches to guarantee that the total electric load is shared between the sources in a desirable manner. A novel communication channel based secondary control method is proposed in this paper. Stability of the proposed method is investigated and it proves that the system stability margin is upgraded using the compensation method. The analytical results of the study will be supported by both time-domain simulations and experimental results.
\end{abstract}

\section{Introduction}

More electric aircraft (MEA) concept is a major trend in modern aerospace engineering. The MEA will benefit from the reduced maintenance cost and increased reliability by putting more emphasis on the utilization of electrical power instead of hydraulic and pneumatic power [1]. More dc distribution systems are emerging into power grids due to the increasing power electronic interfaced converters. Compared to the ac distribution system, the dc power system has some advantages such as lower cost, higher efficiency and easy integration with renewable energy sources and energy storage devices [2], [3]. DC distribution systems also have been widely accepted in transportation electrical systems due to the aforementioned advantages [4], [5]. The sources in a distributed control scheme operate cooperatively to regulate the bus voltage, but a load sharing problem arises, where each source must provide power to the load proportional to its power capacity. In such a context, load sharing is critical to avoid that some sources become overloaded, losing the reliability of distributed power systems. Appropriate power sharing among the sources is of importance in multi-source configuration.

Page 1 of 10
As a decentralized control method, droop control has been widely accepted since no communication among the sources is needed, which improves the system reliability and reduces cost. The core concept of the droop characteristic is to inject desired power/current to the dc bus by voltage drop. As discussed in [7]-[9], there is a tradeoff between droop coefficients and voltage regulation. High droop gain can guarantee precise power sharing among the sources while the voltage regulation performance is poor, i.e., voltage drop is high under high droop gains. In [10], a three level control structure is proposed so that good load sharing and voltage regulation can be achieved. Secondary control is a voltage PI control used to solve the voltage deviation owing to voltage droop. An enhanced droop method with improved voltage regulation is proposed in [11]. However, the communications between the modules are still needed to generate desired compensation values. PI controllers are needed to control the average voltage and current. In [12], large droop gain is recommended to overcome the load sharing error caused by line resistance and average current is used to modify every droop characteristic line so that every droop line will be shifted up a bit by a same amount with the increase of the load. However, the average current needs to be computed and the chosen of shift gain becomes important which is not easily implemented in reality. In [13], a unified compensation method is proposed using the common load condition to compensate the voltage drop. However, the selection principle of compensation coefficient is also not easy. Furthermore, the average current value in per unit needs to be calculated which adds some burden for software implementation.

In this paper, an improved secondary control method is proposed to restore the bus voltage in the droop-controlled system. It is easily implemented since no extra controllers are needed to eliminate the bus voltage deviation. Stability of the proposed method is investigated and it proves that the system stability margin is upgraded using the compensation method. Simulation and experimental validation confirms the effectiveness and performance of the proposed secondary control method.

\section{System Modelling}

Figure 1 shows the generalized dc distribution system with multiple parallel sources. The main dc bus is powered by variable frequency sources $\left(G_{1}-G_{n}\right)$ controlled by pulse-width modulated (PWM) converters $A R_{1}-A R_{n}$ correspondingly. Prime sources can be utility grid, generators, and etc. In Figure $1 C_{1}-C_{n}$ correspond to the local converter output capacitors (local buses) and $C_{b}$ is a capacitor bank installed on the main dc bus. The load sharing among the sources is 
achieved by implementing dc voltage droop characteristics. Load shown in Figure 1 represents the accumulative load including resistive load and power electronic interfaced converters or motor drives.

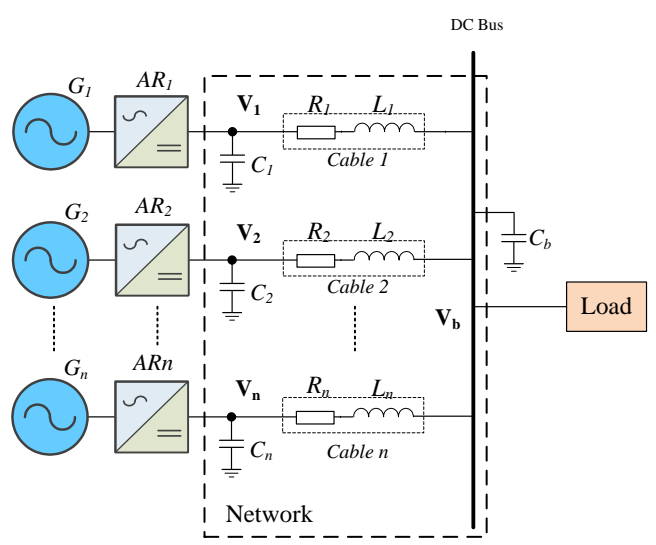

Figure 1. Configuration of DC EPS in the MEA.

Depending on the control strategy, the converters can be controlled either as a voltage source or a current source. The current-mode droop control scheme is shown in Figure 2 with the current reference derived from the specified I-V droop characteristic based on the dc voltage measurement. The target of current-mode system is to control the dc current to follow the reference value computed from droop characteristic shown below,

$$
I_{d c}^{*}=\frac{V_{o}-V_{d c}}{k}
$$

where $V_{o}$ is the nominal voltage $(270 \mathrm{~V})$ in the example $\mathrm{dc}$ distribution system; $k$ is the droop gain; $V_{d c}$ is the terminal dc voltage measurement; $I_{d c}{ }^{*}$ is the generated dc current reference.

On the other hand, the control scheme for voltage-mode droopcontrolled AR is shown in Figure 3. As expressed in (2), the dc voltage reference is generated according to the branch output dc current using V-I droop characteristic.

$$
V_{d c}^{*}=V_{o}-k I_{d c}
$$

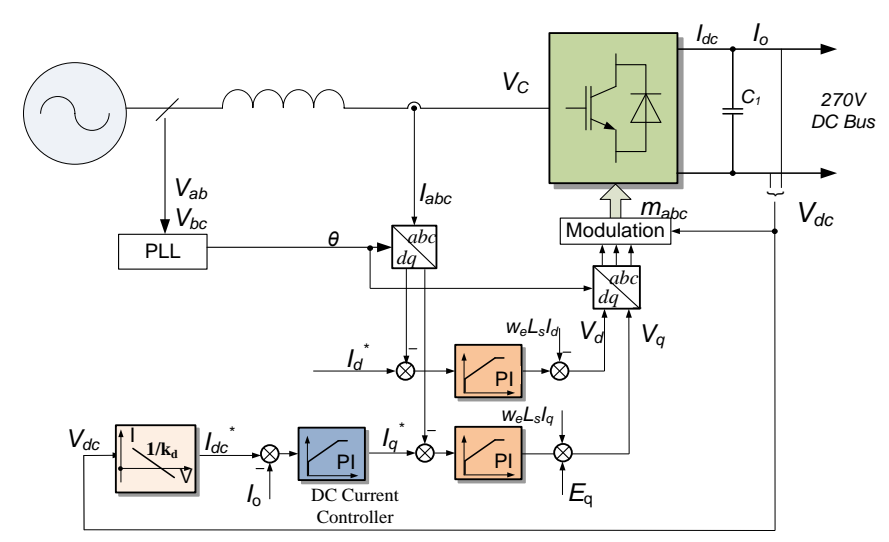

Figure 2. Control schemes for current-mode droop-controlled AR.

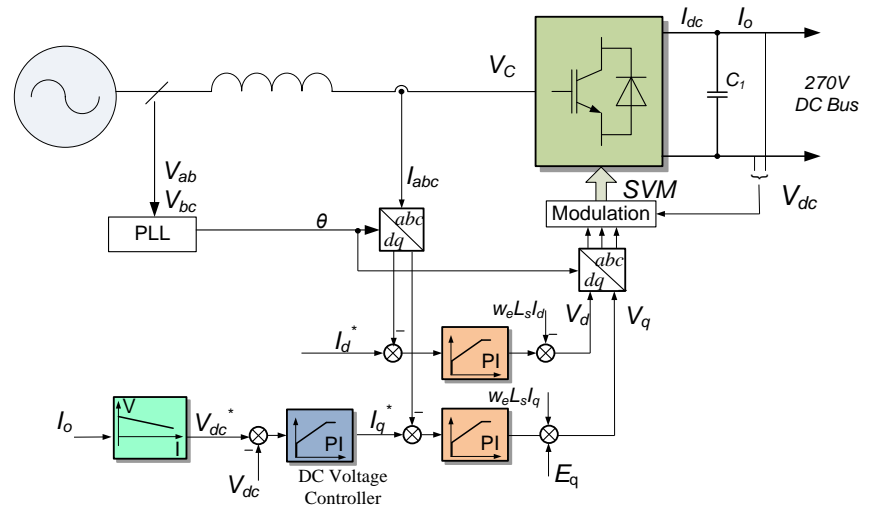

Figure 3. Control schemes for voltage-mode droop-controlled AR.

The two major problems in droop-controlled system are establishing desirable load sharing ratio accuracy between the sources, and ensuring the appropriate voltage regulation. Current (load) sharing in steady state is given by (3):

$$
\frac{I_{i}}{I_{1}}=\frac{k_{1}}{k_{i}}=\frac{n_{i}}{n_{1}}
$$

where $n_{\mathrm{i}}$ is the weighting proportion of the $i$ th source current $I_{\mathrm{i}}$ with respect to the 1 st source current $I_{1}, R_{\mathrm{i}}$ is the $i$ th cable resistance, $k_{\mathrm{i}}$ is the droop gain. In practice, this ratio is affected by cable impedances as shown in (4).

$$
\frac{I_{i}}{I_{1}}=\frac{k_{1}+R_{1}}{k_{i}+R_{i}}=\frac{n_{i}}{n_{1}}
$$

It can be seen from (4) that the accuracy of load sharing will be deteriorated by the cable impedance. In order to mitigate the adverse effect of cable influence, two approaches can be employed here. One is to modify the droop gain according to the actual cables resistances as follows:

$$
k_{i}=\frac{n_{1}}{n_{i}}\left(k_{1}+R_{1}\right)-R_{i}
$$

However, this approach will require knowledge (measurement) of the cable impedance. Taking into account that the cable resistance is not constant during EPS operation and highly depends on environmental conditions, this approach faces certain practical limitation.

An alternative solution is to set a relatively large droop gain $\left(k_{\mathrm{i}}>>R_{\mathrm{i}}\right)$ such that the impact of R-terms in (4) becomes negligible. The current sharing accuracy will be improved however the voltage regulation will be high and unacceptable for some applications. For example, MEA EPS are subject of power quality standard MIL-STD704F [14]: for 270V DC system the voltage.

The voltage deviation at the main bus would be high under heavy load condition if a soft droop slope is applied. Sometimes, high voltage drop will violate the voltage requirement at steady state. As illustrated in MIL_STD_704F [14], steady-state limits for dc voltage in the $270 \mathrm{~V} \mathrm{dc}$ system is between $250 \mathrm{~V}$ and $280 \mathrm{~V}$. Therefore, voltage compensation is of importance for the system and proportional power sharing should be achieved simultaneously, especially at heavy load condition. 


\section{Proposed Secondary Control Method}

\section{A. Working Principle}

The working principle of the secondary control method is illustrated in Figure 4. At any load condition, the voltage deviation $(\Delta V)$ using the conventional droop characteristic is compensated. Correspondingly the slope is kept as before but the terminal voltage is restored to its nominal value.

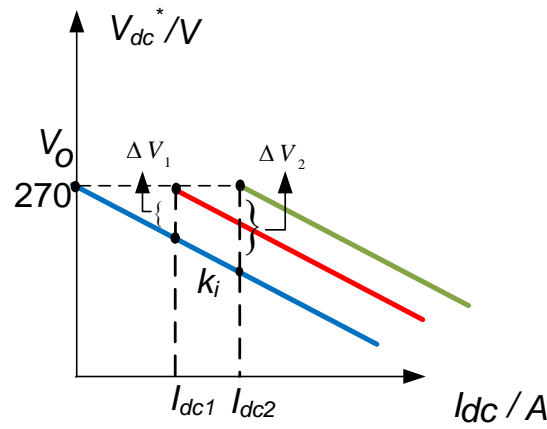

Figure 4. Working principle of the secondary control.

In order to reduce the voltage drop under severe load condition, the paper proposes an enhanced secondary control approach shown in Figure 5 for the current-mode droop-controlled system and in Figure 6 for the voltage-mode droop-controlled system, respectively. A communication line is employed to ensure the same compensation level for each module. A feedforward term $\left(\Delta V_{\mathrm{j}}\right)$ which is added to the nominal voltage of each module can be expressed as follows:

$$
\begin{gathered}
\Delta V_{j}=\frac{\sum_{i=1, i \neq j}^{i=n} I_{i} k_{i} G_{d}+I_{j} k_{j}}{n} \\
G_{d}=\frac{1}{T_{d} s+1}
\end{gathered}
$$

where $n$ is the number of active modules in the system, $G_{d}$ is the transfer function of the communication channel; $T_{\mathrm{d}}$ is the delay time of the communication channel.

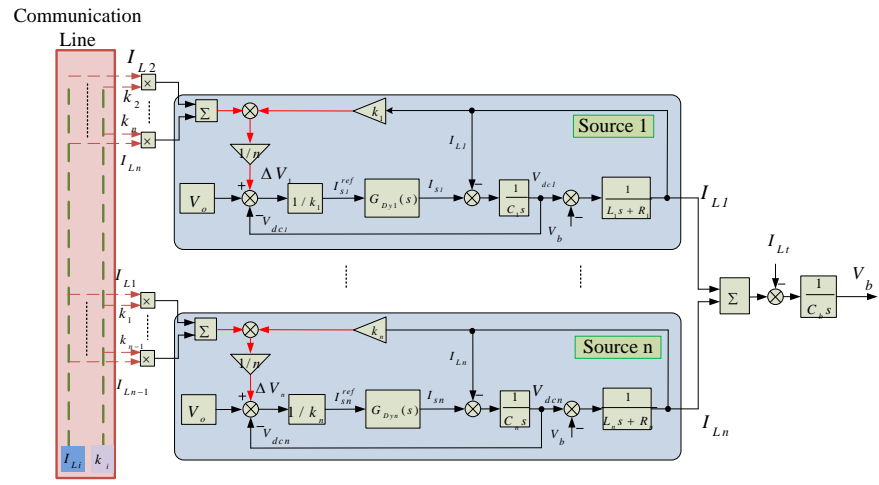

Figure 5. Proposed secondary control approach for the current-mode droopcontrolled system.

Page 3 of 10

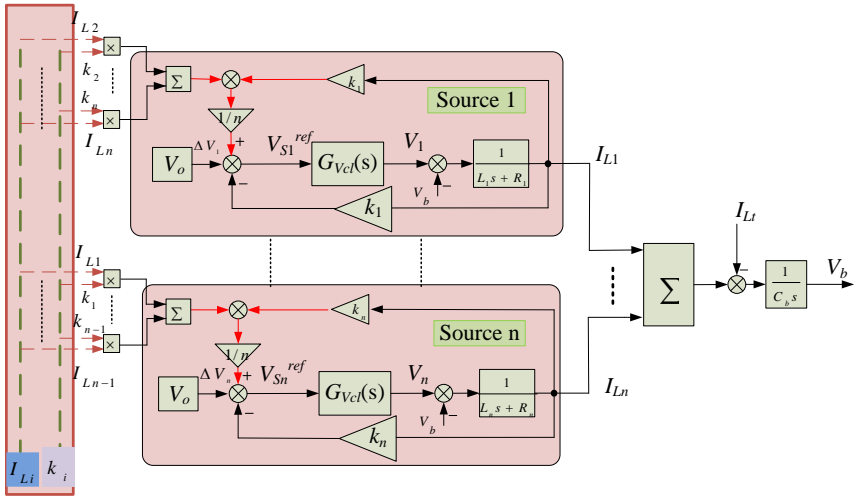

Figure 6. Proposed secondary control approach for the voltage-mode droopcontrolled system.

Similar to the individual gain set in each parallel module, the V-I characteristic at the main bus can be expressed as a linear curve with a constant slope. It is called global droop gain afterwards in the paper.

Bus voltage can be expressed using the KVL as follows:

$$
\begin{aligned}
V_{b} & =V_{o}-I_{1}\left(k_{1}+R_{1}\right)=V_{o}-I_{2}\left(k_{2}+R_{2}\right) \\
& =\ldots=V_{o}-I_{n}\left(k_{n}+R_{n}\right)
\end{aligned}
$$

Therefore, the total load current can be written in terms of the main bus voltage in (9),

$$
I_{L}=I_{1}+I_{2}+\ldots+I_{n}=\left(V_{o}-V_{b}\right) \sum_{i=1}^{n} \frac{1}{k_{i}+R_{i}}
$$

The main bus voltage can be reframed as a function of total load current and individual droop gain coefficients,

$$
V_{b}=V_{o}-I_{L} \frac{1}{\sum_{i=1}^{n} \frac{1}{k_{i}+R_{i}}}
$$

After the V-I relationship is obtained by (10), the global droop gain $k_{t}$ can be expressed as follows,

$$
k_{t}=\frac{1}{\sum_{i=1}^{n} \frac{1}{k_{i}+R_{i}}}
$$

where $R_{i}$ is the cable resistance. This global droop coefficient determines the main bus V-I characteristic. 


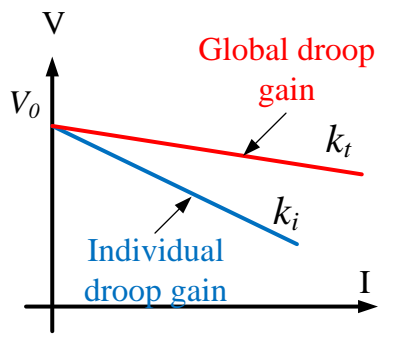

Figure 7. Relationship between global droop gain and individual droop gain.

The relationship between global droop gain and individual droop gain is depicted in Figure 7. It can be seen that the global droop slope is stiffer than individual droop gain. In other words, under the same load power, the voltage drop at the main bus with multi-source operation is smaller than the voltage deviation under single source operation scenario.

\section{B. Normal Scenario}

The state-space model has been established. Based on these, the eigenvalues locus of the proposed secondary control method and conventional droop control method is illustrated in Figure 8. Different global droop gain is tested as shown in Figure 8(a) and (b). It is observable that the dominant eigenvalues of the system will move towards left when the proposed method is activated which indicates the system is more stable using the proposed method. It can also be explained in the view of impedance. After the proposed method is activated, the magnitude of load impedance is increased since the bus voltage restores to nominal value. Since the source impedance does not change accordingly, the stability margin is increased using the proposed compensation method.

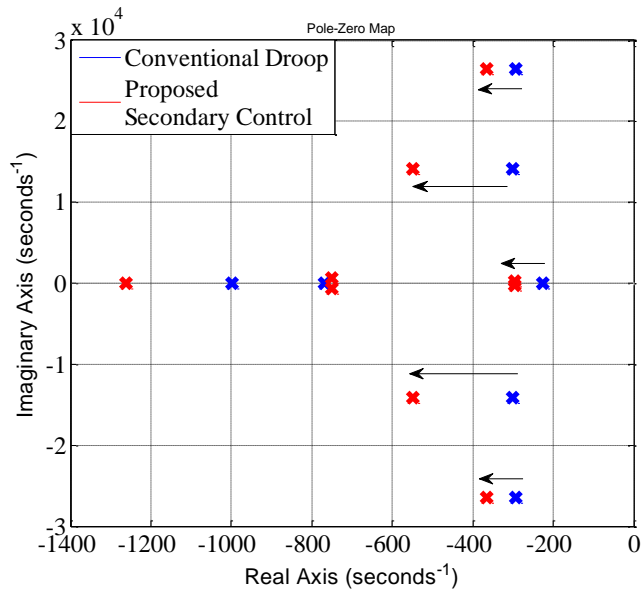

(a)

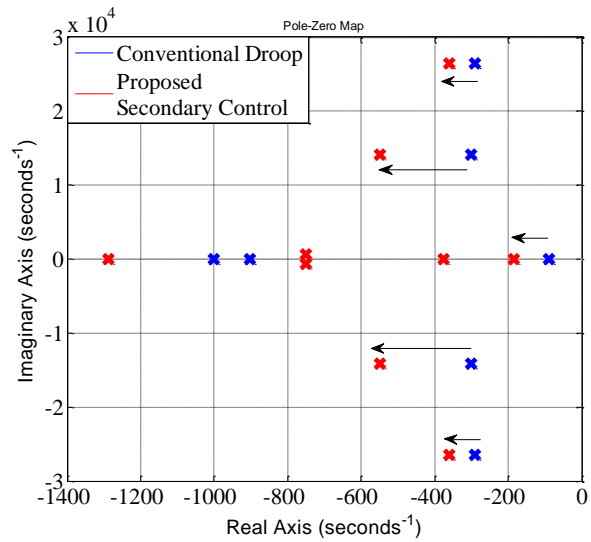

(b)

Figure 8. Dominant eigenvalues movement for the proposed secondary control method. (a) $k_{\mathrm{t}}=2$. (b) $k_{\mathrm{t}}=4$.

\section{Fault Scenario}

For normal scenarios, when the secondary control is activated, terminal voltage will restore to nominal voltage at steady-state for any load current. If one or multiple contingency occurs, the other sources will share the load power according to their individual droop constant. The proportional power sharing is still ensured under this circumstance. When the contingency happens for one or multiple sources in the system, the number of active modules will be updated and the compensation term $\left(\Delta V_{\mathrm{j}}\right)$ will change accordingly to realize the voltage restoration.

If the number of active modules is not updated in one communication process, based on Figure 5 and Figure 6 a smaller compensation term will be added to each parallel modules and as a result, the dc bus voltage is not restored to its nominal voltage but to a smaller value. Hence, compared to the droop-controlled system without the proposed secondary control approach, the voltage regulation is still improved. It will be demonstrated in the subsequent experimental results.

When the number of active modules is updated, the bus voltage deviation will be eliminated and it improves the power quality to meet the bus voltage specifications.

Overall, using the proposed approach, the bus voltage will restore to its nominal value or a smaller value. Thus, it is worth noting that the proposed secondary control approach will not result in over-voltage at the main dc bus.

In summary, the proposed secondary control approach could improve the bus voltage regulation even under fault scenarios. 


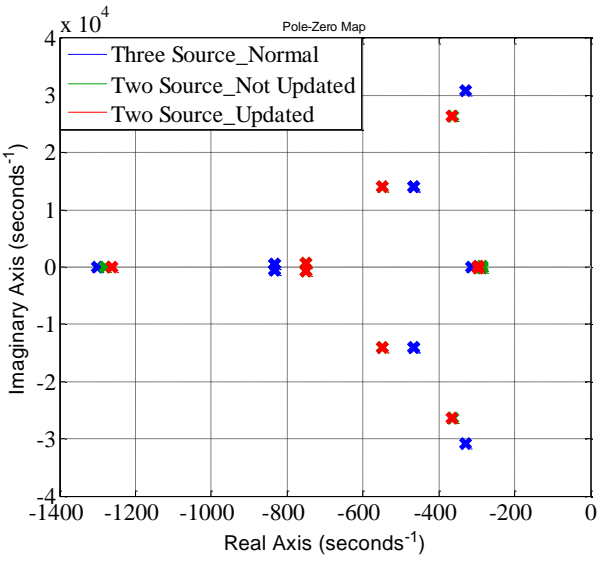

Figure 9. Fault scenario with the proposed secondary control method (Outage of one source)

\section{Effect of Communication Delay}

The effect of the communication delay $T_{\mathrm{d}}$ on previous communication channel based secondary control methods are discussed in [16]-[18]. In this subsection, the impact of the communication delay on the stability is analyzed using eigenvalue analysis. As shown in Figure 10, the eigenvalues contour shows that a proper communication delay should be selected to ensure system stability. For power sharing ratio 1:5 case (see Figure 10(a)), it can be seen that a pair of eigenvalues are located in the RHP when 1 us communication delay is applied, which indicates that the system with 1 us communication delay is unstable. However, the communication channel delay can easily reach the values in the order of milliseconds or even tens of millliseconds [17]. It can be seen that system can be stabilized using $0.1 \mathrm{~s}, 1 \mathrm{~ms}$ communication delay. For the equal sharing case shown in Figure 10(b), it can be seen that all eigenvalues are still in the left of the $\mathrm{S}$ domain with 1 us delay. It confirms again that the unequal sharing case degrades the stability margin compared to the equal sharing case.

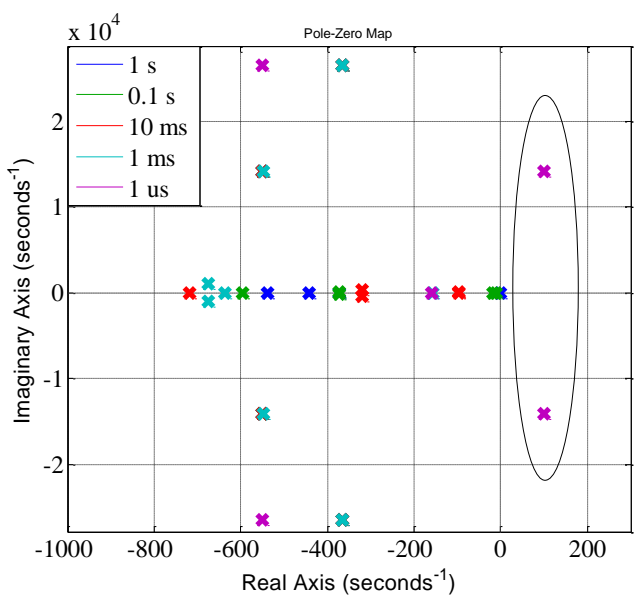

(a)

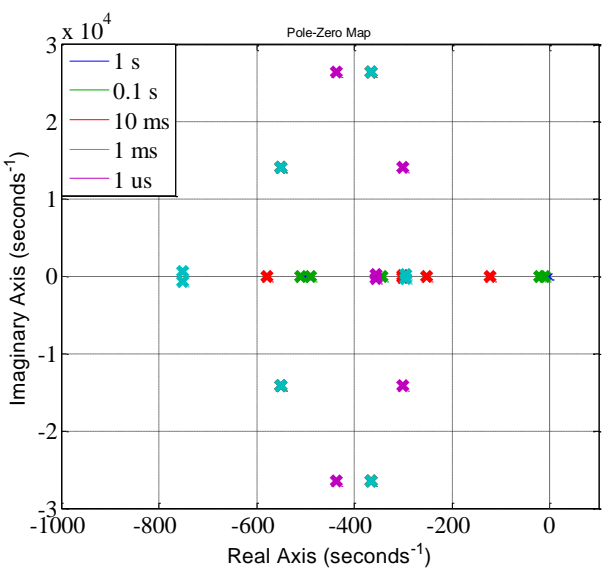

(b)

Figure 10. Dominant eigenvalues with respect to the communication delay in two VSCs paralleled system under different power sharing ratio. (a) 1:5; (b) $1: 1$

\section{E. Effect of Power Sharing Ratio}

Different load sharing ratio may have different stability margins. It is shown in Figure 11 that the dominant eigenvalues move towards RHP as the ratio between the two modules increases, which indicates that the discrepancy of the power sharing ratio will degrade the system stability.

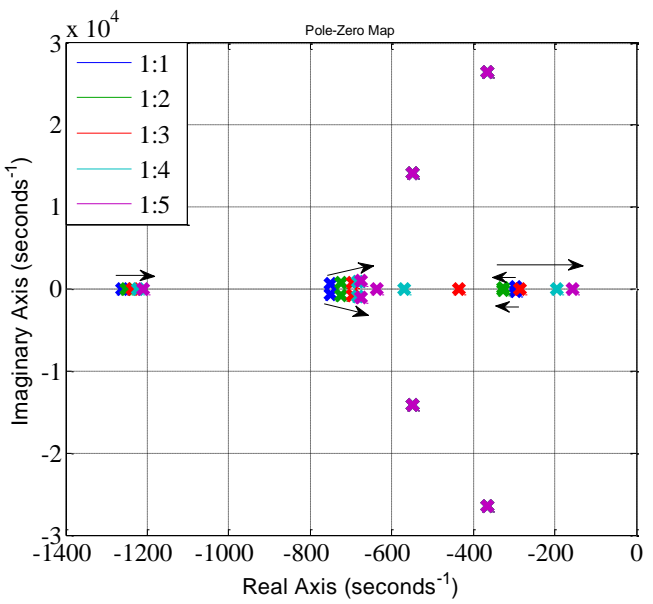

(b)

Figure 11. Dominant eigenvalues contours with respect to different power sharing ratios (1 $\mathrm{ms}$ communication delay).

\section{Simulation Studies}

To support the analytical analysis in the previous Sections, a potential DC EPS with twin power converters working in parallel was modelled in Matlab/Simulink. A DC/DC converter is tightly controlled as a constant power load (CPL). The topology shown in Figure 12 considered in this section can be viewed as a fundamental subsystem of more complex MEA EPS. The EPS parameters are listed in Table 1 and the simulated scenarios are given in Table 2. 


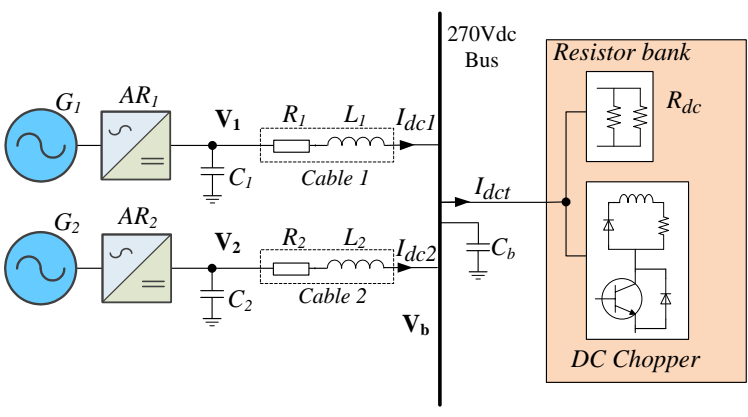

Figure 12. Simulation topology.

Table 1. Simulation system parameters

\begin{tabular}{|c|c|c|}
\hline Parameter & Symbol & Value \\
\hline Nominal voltage & $V_{o}$ & $270 \mathrm{~V}$ \\
\hline Local shunt capacitor & $C_{i}$ & $1 \mathrm{mF}$ \\
\hline Cable inductance & $L_{i}$ & $1 \mu \mathrm{H}$ \\
\hline Cable resistance & $R_{i}$ & $0.2 \Omega$ \\
\hline Resistive load & $R_{\text {res }}$ & $47 \Omega$ \\
\hline
\end{tabular}

Table 2. Simulation event

\begin{tabular}{|c|c|}
\hline Time (s) & Events \\
\hline 0.2 & $+0.5 \mathrm{~kW}$ CPL applied \\
\hline 0.4 & $+0.5 \mathrm{~kW}$ CPL applied \\
\hline 0.6 & $+1 \mathrm{~kW}$ CPL applied \\
\hline 0.8 & Resistive load disconnected \\
\hline 1 & Resistive load reconnected \\
\hline
\end{tabular}

\section{A. Normal Scenario}

If a small droop gain is applied, the voltage drop at the main bus is small even at heavy loads due to stiff global droop characteristic. However, the current sharing ratio is not exactly $2: 1$ as desired because the cable resistances influence the accuracy of the current sharing, according to (4). Therefore, the individual droop gains were set to 3 and 6 respectively to satisfy the condition $k_{i} \gg R_{c}$. The global droop gain, according to (11), becomes equal to 2 . Figure 13 shows the simulation results based on these settings. The dashed lines show the results when the proposed compensation method is employed. It can be seen from Figure 13(a) that the current sharing ratio is close to $2: 1$. The proportional load sharing is guaranteed under the proposed compensation method. The performance of current sharing is also good when the load sheds as confirmed by scenario when the resistive load switched off ( $t=0.8 \mathrm{~s})$ and then switched on at $t=1 \mathrm{~s}$. After $t=0.6 \mathrm{~s}$, the current of each module is reduced and consequently, the line losses are reduced when the proposed compensation method is applied. As shown in Figure 13(b), the bus voltage in steady-state is kept at rated value.

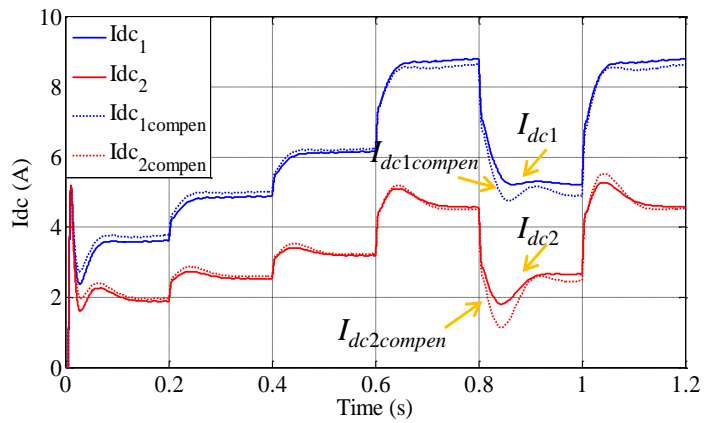

(a)

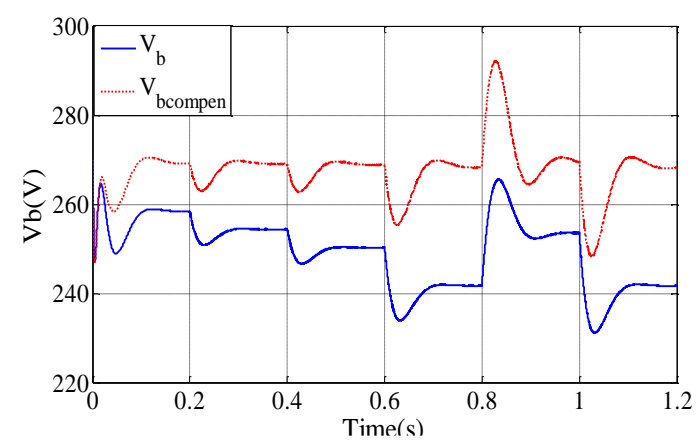

(b)

Figure 13. Simulation results of the parallel system $\left(k_{1}=3, k_{2}=6\right)$. (a) DC current waveforms. (b) Bus voltage waveforms.

Figure 14 shows the DC current and bus voltage when individual gain is set to 4 for both converters. The global droop gain is still 2 as previous case. It can be seen from Figure 14(a) that the equal current sharing between the two modules is achieved no matter whether the compensation method is activated. Similar to the results in Figure 13, the transmission line losses is decreased after CPL hits $2 \mathrm{~kW}$.

\section{B. Fault Scenario}

A source fault scenario was simulated to test the robustness of the proposed method. Prior to $t=1.2 \mathrm{~s}$, both converters are working in parallel to share the load power equally with the identical individual droop gains $\left(k_{1}=k_{2}=4\right)$. It can be seen from Figure 15 that the converter 1 will take the full responsibility of providing power to meet the load demand after the loss of converter 2 at $t=1.2 \mathrm{~s}$. Meanwhile, the compensation method is still active and compensation gain is not updated in real time. The bus voltage drops from nominal voltage to $240 \mathrm{~V}$ at steady state, indicating that the proposed method still compensates the bus voltage drop to some extent but cannot fully compensate the voltage deviation since the global droop gain under new EPS conditions is not updated. When the proposed compensation method is deactivated after $t=1.6 \mathrm{~s}$ (conventional droop scheme), the bus voltage will further drop to 212 $\mathrm{V}$ in steady state. This simulation confirms that the proposed compensation method is robust and performs well under the fault scenario. It is in accordance with the analysis in Section III-C. Even if the compensation is not real-time updated after the contingency occurs, the voltage deviation can still be reduced compare to the case without the proposed approach. 


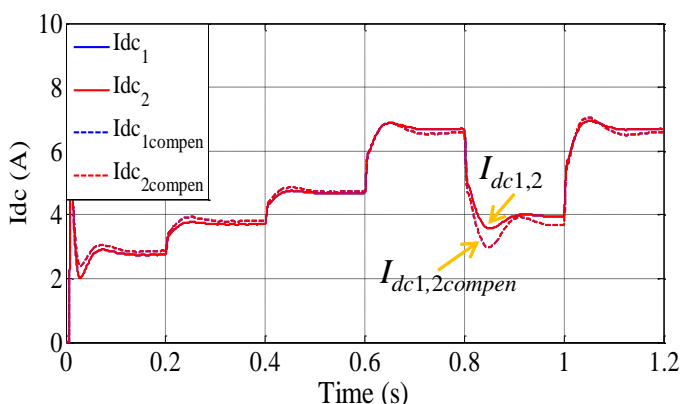

(a)

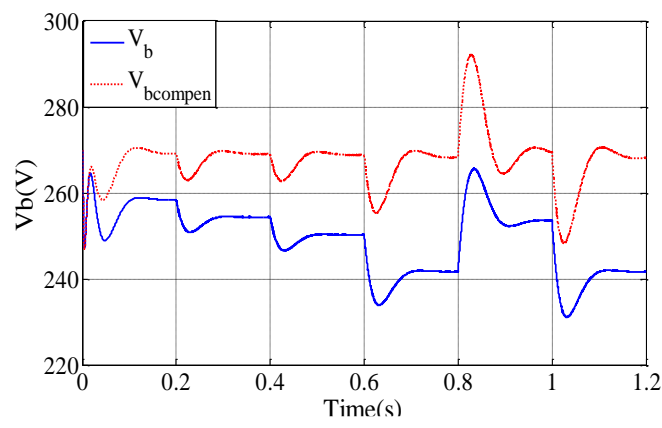

(b)

Figure 14. Simulation results of the parallel system $\left(k_{1}=k_{2}=4\right)$. (a) DC current waveforms. (b) Bus voltage waveforms.
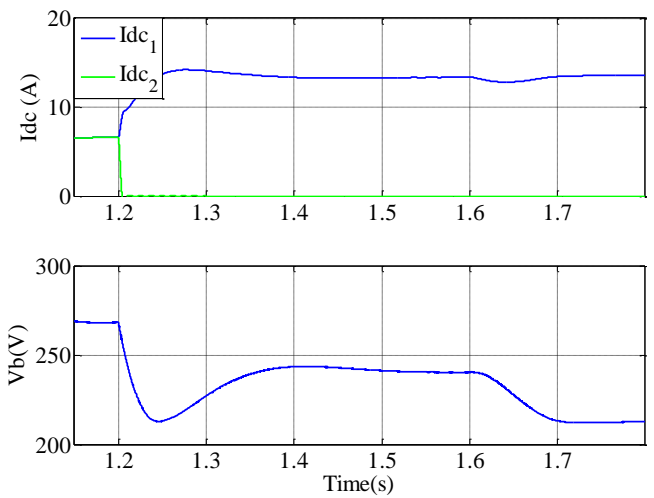

Figure 15. Simulation results at fault scenario.

\section{Effect of Communication Delay}

In this subsection, only CPL is used to mimic the worst case in terms of stability. Global droop gain is set to 2 and the load sharing ratio is $1: 8$. CPL increases step-wise at $\mathrm{t}=0.2 \mathrm{~s}, 0.5 \mathrm{~s}$ and $0.8 \mathrm{~s}$ respectively. Figure 16 presents the impact of communication delay on system stability. It is shown in Figure 16 (a) that the output current from converter 1 and 2 satisfies the desired ratio 1:8 at any load condition. Further, the system stability is guaranteed even at high CPL condition. However, it can be seen from Figure 16 (b) that system with 1 us delay will go unstable. The power required by the CPL exceeds the power limit and the system collapses. The bus voltage drops very fast Page 7 of 10 and the buck converter tries to provide the power demanded by the load modifying the duty cycle for increasingly higher values, until the duty cycle value saturates, forcing the switch to remain closed. At this point, the buck converter loses its constant power characteristic and starts to behave passively as an RLC circuit. That is why the system finds another equilibrium point.
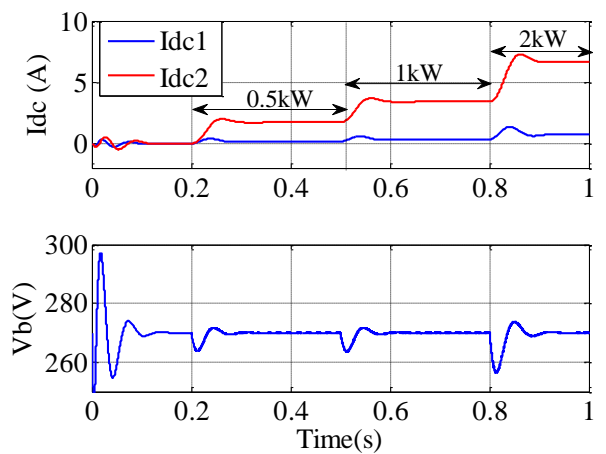

(a)
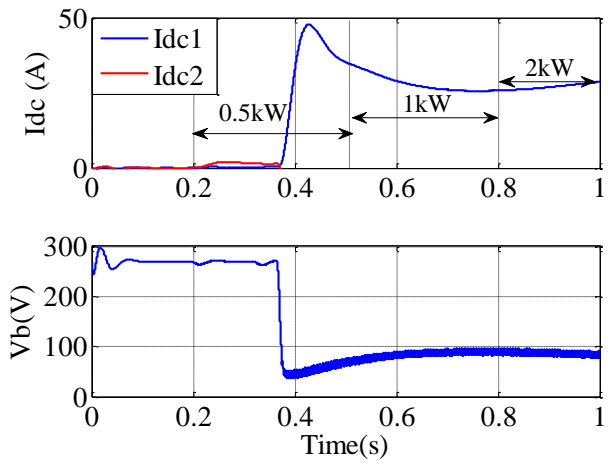

(b)

Figure 16. Simulation results for proposed compensation method with different load sharing $\left(k_{1}=9 / 4, k_{2}=18\right)$. (a) $T_{\mathrm{d}}=1 \mathrm{~ms}$; (b) $T_{\mathrm{d}}=1 \mathrm{us}$.

Compared with the unequal power sharing case shown in Figure 16, the individual droop gain is set to be equal and the global droop gain is still 2. Figure 17 shows that system with equal sharing ratio is stable with 0.1 us communication line. In contrast with Figure 16(b), it indicates that the equivalent load sharing among the sources has more stability margin than inequivalent sharing.
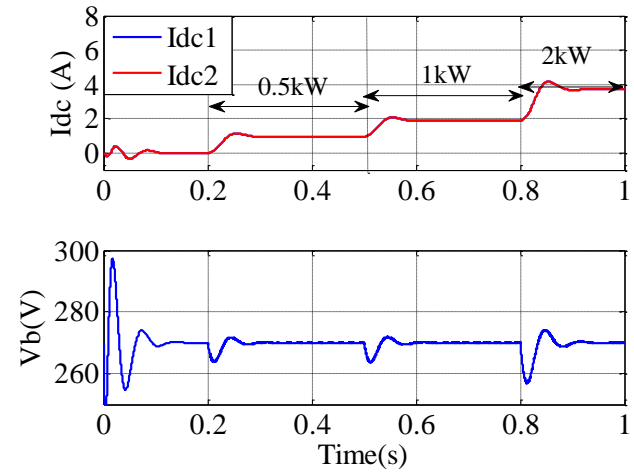

Figure 17. Simulation results for proposed compensation method with equal load sharing. 


\section{Experimental Results}

A prototype EPS consisting of two parallel active front-end converters (Semikube) has been constructed, as shown in Figure 18 to validate the performance of proposed voltage compensation method. PC1 and PC2 are utilized to control two converters (Conv 1, Conv 2) separately. A DC/DC converter (buck converter) with resistor is tightly regulated in constant power mode. The experimental system parameters are listed in Table 3 . The three-phase input voltage for each module is isolated through a step down transformer $(415 \mathrm{~V} / 160 \mathrm{~V})$ in which primary side is connected to the $415 \mathrm{~V}$ (line-to-line RMS voltage) utility grid.

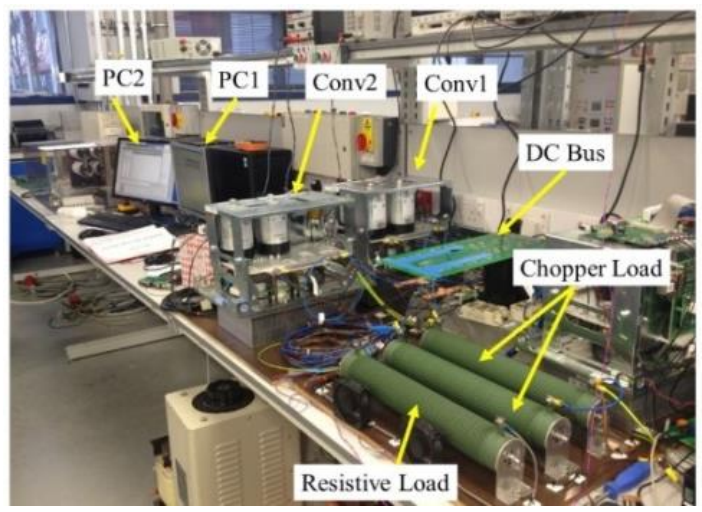

Figure 18. Experimental setup.

Table 3. Experimental system parameters

\begin{tabular}{|l|l|c|}
\hline Category & Parameter & Value \\
\hline Three phase grid & Grid source voltage & $415 \mathrm{~V}$ line-to-line RMS \\
\hline \multirow{2}{*}{ Transformer } & $\begin{array}{l}\text { Primary/Secondary } \\
\text { voltage }\end{array}$ & $415 \mathrm{~V} / 160 \mathrm{~V}, \mathrm{Y}-\mathrm{y}$ \\
\cline { 2 - 3 } & Ratings & $20 \mathrm{kVA}$ \\
\hline \multirow{2}{*}{ Active Rectifier } & Switching frequency & $10 \mathrm{kHz}$ \\
\cline { 2 - 3 } & Local capacitor & $1.2 \mathrm{mF}$ \\
\hline Resistive Load & Resistance & $47 \Omega$ \\
\hline DC/DC Converter & Chopper load & $23.5 \Omega$ \\
\hline \multirow{2}{*}{ DC Link } & DC link capacitor & $0.8 \mathrm{mF}$ \\
\cline { 2 - 3 } & DC link bus voltage & $270 \mathrm{~V}$ \\
\hline \multirow{2}{*}{ Cable } & Line resistance & $200 \mathrm{~m} \Omega$ \\
\cline { 2 - 3 } & Line inductance & $1 \mu \mathrm{H}$ \\
\hline
\end{tabular}

\section{A. Unequal Sharing Case (Case 1)}

Initially the individual droop gain for Conv 1 and Conv 2 is 3 and 6 , respectively. Figure 19(a) shows the bus voltage and DC current of the test rig with CPL $(2 \mathrm{~kW})$ and $47 \Omega$ resistor using conventional droop control method (see Fig. 2). It can be seen that DC currents injected to the main bus are $8.8 \mathrm{~A}$ and $4.35 \mathrm{~A}$ respectively which satisfies the desired ratio 2:1. After the proposed voltage compensation method is activated (see Figure 19(b)), the main bus voltage has recovered to $268 \mathrm{~V}$. The current sharing ratio between the two converters is still $2: 1$, whilst the converters currents are reduced to $8.3 \mathrm{~A}$ and $4.15 \mathrm{~A}$, respectively. The practical result agrees with the transmission loss-based analysis in Section IV.

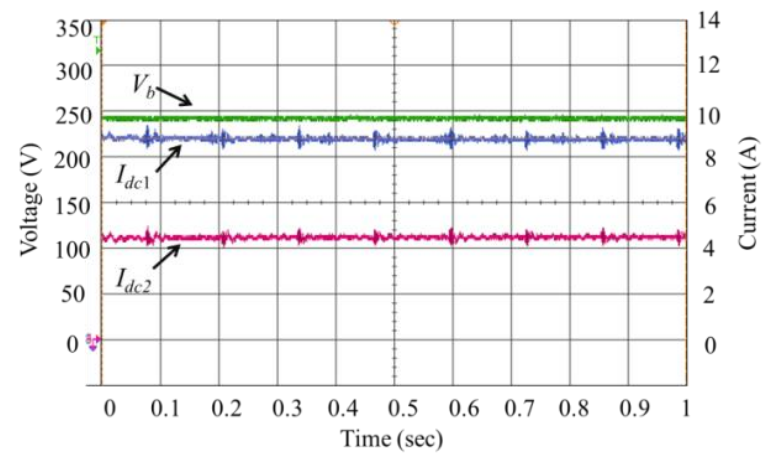

(a)

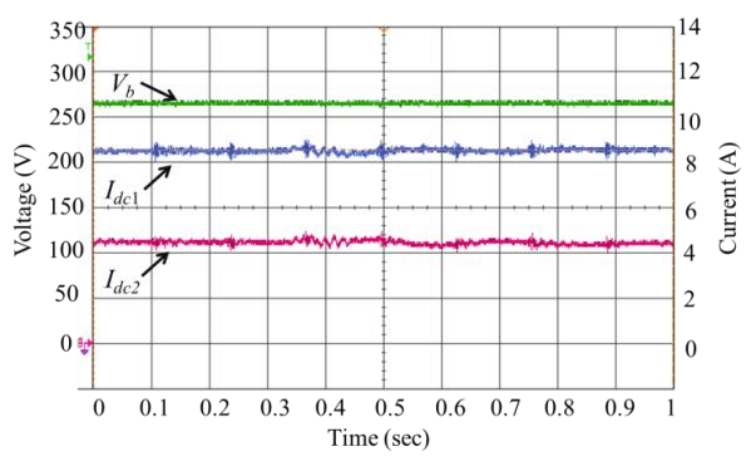

(b)

Figure 19. Experimental result of the unequal sharing case $\left(k_{1}=3, k_{2}=6\right)$. (a) without proposed secondary control, (b) with proposed secondary control.

\section{B. Equal Sharing Case (Case 2)}

In the equal sharing case, the global droop gain at the main bus is still set to 2 , but the individual droop gains are set to 4 for each converter. Thus, the current ratio of the two converters is expected to be $1: 1$.

Figure 20(a) shows the experimental results of the system under conventional droop control. The current for each module is $6.8 \mathrm{~A}$. The bus voltage is still $240 \mathrm{~V}$, which is identical to Case 1 . After the proposed method is implemented, it can be seen from Figure 20(b) that bus voltage recovers to $268 \mathrm{~V}$. The branch current for each module is reduced to $6.5 \mathrm{~A}$. This result is consistent with the theoretical analysis, the proposed restoration method facilitates reducing the transmission losses.

\section{Fault Scenario}

The feasibility of using the proposed voltage compensation method under faulty conditions is demonstrated in Figure 21. Prior to $t=t_{1}$, both converters are operated in parallel and the proposed voltage restoration method is activated. At $t=t_{1}$, the outage of Conv 2 occurs and as a consequence Conv 1 takes the full responsibility to feed the load. Between $t=t_{1}$ and $t_{2}$, the global droop gain is not updated, thus

Page 8 of 10 
the main bus voltage drops to $261.6 \mathrm{~V}$. The updated global droop gain is used for the working converter (Conv 1 ) at $t=t_{2}$, it is seen that the main bus voltage recovers to approximately $270 \mathrm{~V}$. At $t=t_{3}$, the proposed compensation method is deactivated and the bus voltage reduces further to $253.3 \mathrm{~V}$. these results confirm that the proposed restoration approach can effectively reduce the voltage deviation under faulty condition even if the global droop gain cannot be updated in time.

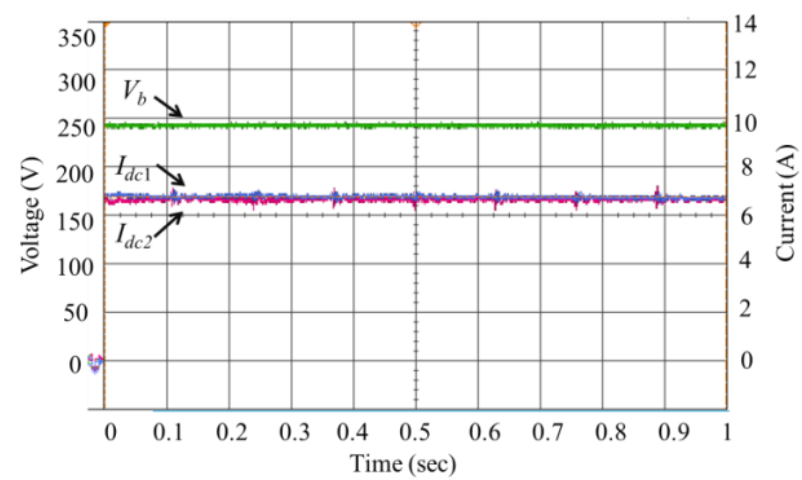

(a)

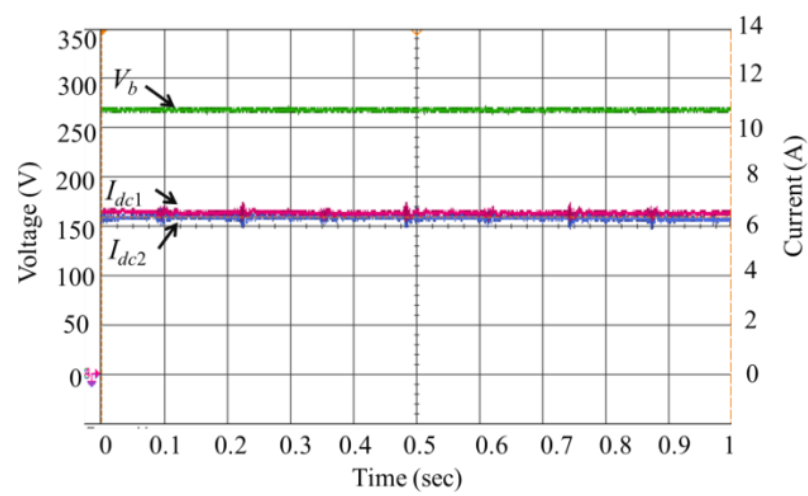

(b)

Figure 20. Experimental result of the equal sharing case $\left(k_{1}=k_{2}=4\right)$. (a) without proposed secondary control, (b) with proposed secondary control.

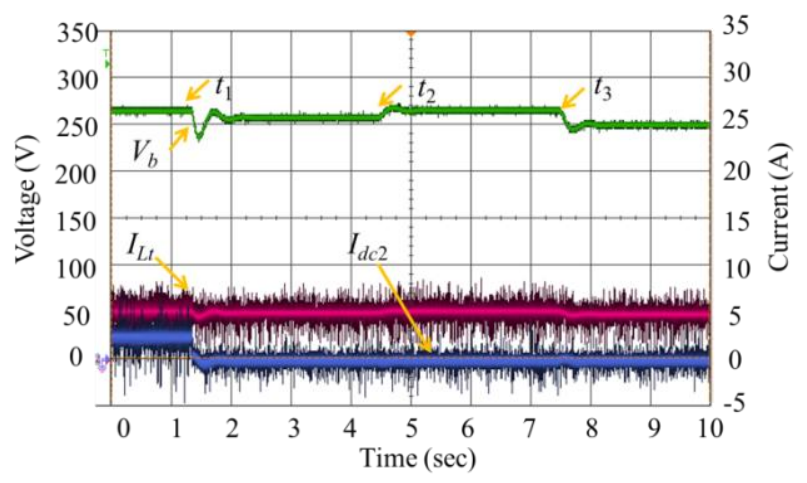

Figure 21. Experimental result for fault scenario.

\section{Conclusions}

In this paper, different droop control strategies (current-mode/voltage mode) are reviewed. The power system architecture assumed twin sources controlled by power electronics running in parallel under different types of droop control (current-mode and voltage-mode) feeding the mix load (conventional linear load and constant power load, CPL). It is shown that the application of traditional drooping strategies may result in poor bus voltage regulation and even deviate voltage specifications in MIL-STD-704F. The study proposed a novel secondary control method allowing simultaneous satisfying requirements of both power sharing accuracy and voltage control using analytical solutions derived in the paper. Stability analysis of generalized EPS under the proposed droop was analyzed and reported. It was shown that in the case of a multi-feed EPS, the stability boundaries are not only dependent on traditionally considered parameters (CPL power, bus capacitances etc) but also dependent on the load sharing ratio among the sources.

Employing the proposed method, the system can work under heavy load condition with the soft droop characteristic and meanwhile the power sharing among the parallel sources are guaranteed. The performance and robustness of the proposed method is validated by experimental results as well.

\section{References}

1. Rosero J. A., Ortaga J. A., Aldabas E., and Romeral L., "Moving towards a more electric aircraft," IEEE Aerosp. Electron. Syst. Mag., vol. 22, no. 3, pp. 3-9, Mar. 2007.

2. N. Eghtedarpour and E. Farjah, "Power control and management in a hybrid ac/dc microgrid," IEEE Trans. Smart Grid, vol. 5, no. 3, pp.1494-1505, May 2014.

3. S.-M. Chen, T.-J. Liang, and K.-R. Hu, "Design, analysis, and implementation of solar power optimizer for dc distribution system," IEEE Trans. Power Electron., vol. 28, no. 4, pp. 1764 1772, Apr. 2013.

4. J. Ciezki and R. Ashton, "Selection and stability issues associated with a navy shipboard dc zonal electric distribution system," IEEE Trans. Power Del., vol. 15, no. 2, pp. 665-669, Apr. 2000.

5. D. Izquierdo, R. Azcona, F. del Cerro, C. Fernandez, and B. Delicado, "Electrical power distribution system (HV270DC), for application in more electric aircraft," in 25th Annu. IEEE Appl. Power Electron. Conf. Expo. (APEC), Canada, Feb. 2010, pp. 1300-1305.

6. Prieto-Araujo, D. F. Bianchi, A. Junyent-Ferre, and O. GomisBellmunt, "Methodology for droop control dynamic analysis of multiterminal VSC-HVDC grids for offshore wind farms," IEEE Trans. Power Del., vol. 26, no. 4, pp. 2476-2485, Oct. 2011.

7. J. M. Guerrero, M. Chandorkar, T.-L. Lee, and P. C. Loh, "Advanced control architectures for intelligent microgrids-Part I: Decentralized and hierarchical control," IEEE Trans. Ind. Electron., vol. 60, no. 4, pp. 1254-1262, Apr. 2013.

8. N. R. Chaudhuri and B. Chaudhuri, "Adaptive droop control for effective power sharing in Multi-Terminal DC (MTDC) grids," IEEE Trans. Power Syst., vol. 28, no. 1, pp. 21-29, Feb. 2013.

9. H. Kakigano, Y. Miura, and T. Ise, "Distribution voltage control for dc microgrids using fuzzy control and gain-scheduling technique," IEEE Trans. Power Electron., vol. 28, no. 5, pp. 2246-2258, May 2013.

10. J. M. Guerrero, J. C. Vasquez, and L. G. D. Vicuna, "Hierarchical control of droop-controlled ac and $\mathrm{dc}$ 
microgrids - A general approach toward standardization," IEEE Trans. Ind. Electron., vol. 58, no. 1, pp. 158-172, Jan. 2011.

11. X. Lu, J. M. Guerrero, K. Sun, and J. C. Vasquez, "An improved droop control method for dc microgrids based on low bandwidth communication with dc bus voltage restoration and enhanced current sharing accuracy," IEEE Trans. Power Electron., vol. 29, no. 4, pp. 1800-1812, Apr. 2014.

12. S. Anand, B. G. Fernandes, and J. M. Guerrero, "Distributed control to ensure proportional load sharing and improve voltage regulation in low voltage dc microgrids," IEEE Trans. Power Electron., vol. 28, no. 4, pp. 1900-1913, Apr. 2013.

13. N. Yang, D. Paire, F. Gao, A. Miraoui, and W. Liu, "Compensation of droop control using common load condition in dc microgrids to improve voltage regulation and load sharing," Int. J. Elect. Power Energy Syst., vol. 64, pp. 752-760, Jan. 2015.

14. Aircraft Electric Power Characteristics, American Military St. MIL-STD-704F, Mar. 2004.

15. N. Jelani and M. Molinas, "Asymmetrical fault ride through as ancillary service by constant power loads in grid-connected wind farm," IEEE Trans. Power Electron., vol. 30, no. 3, pp. 1704-1713, Mar. 2015.
16. P. Wang, X. Lu, X. Yang, W. Wang, and D. G. Xu, "An improved distributed secondary control method for DC microgrids with enhanced dynamic current sharing performance," IEEE Trans. Power Electron., vol. PP, no. 99, pp.1-1.

17. Q. Shafiee, Stefanović, T. Dragičević, P. Popovski, J. C. Vasquez, and J. M. Guerrero, "Robust networked control scheme for distributed secondary control of islanded microgrids," IEEE Trans. Ind. Electron., vol. 61, no. 10, pp. 5363-5374, Oct. 2014.

18. C. Ahumada, R. Cárdenas, D. Sáez, and J. M. Guerrero, "Secondary control strategies for frequency restoration in islanded microgrids with consideration of communication delays," IEEE Trans. Smart Grid, vol. PP, no.99, pp.1-1.

\section{Acknowledgments}

The work is being supported by the Cleansky JTI Project, a FP7 European Integrated Project-http://www.cleansky.eu. 